\title{
TOPSIS Based Multi-radio Access Scheme in Heterogeneous Wireless Networks
}

\author{
Xuewen Zhu ${ }^{\mathrm{a}}$, Qi Zhu ${ }^{\mathrm{b}}$ \\ Jiangsu Key Lab of Wireless Communications, Key Lab on Wideband Wireless Communications \\ and Sensor Network Technology of Ministry of Education Nanjing University of Posts and \\ Telecommunications, Jiangsu Nanjing 210003, China \\ aemail: zhuxw003@163.com, 'email: zhuqi@njupt.edu.cn
}

Keywords: Heterogeneous Wireless Networks; TOPSIS; Multi-radio Access

\begin{abstract}
With the rapid development of various kinds of radio access technology, the trend of future network will be the heterogeneous wireless networks environment, where all kinds of networks coexist for their complementary characteristics. On the other hand, the rise of smart terminal and software defined radio contribute to the popularity of multi-mode terminals (MMTs) that can connect to several networks simultaneously. In this paper, we propose a TOPSIS based multi-radio access algorithm in heterogeneous wireless networks. MMTs determine candidate networks according to received signal strength (RSS) and access networks with better performance in terms of throughput, energy consumption, network load and cost by means of TOPSIS. Simulation results show that the proposed algorithm can enhance the throughput efficiently without consuming too much energy.
\end{abstract}

\section{Introduction}

The rapid development of mobile communication technology and broadband wireless access technology contribute to the increasing number of wireless communication traffic in recent years. Commonly, cellular networks support user mobility and seamless handoff but cost more. While broadband wireless networks charge lower price but only efficient in hotspots. There is not a network can satisfy all users' demands, so that the trend of networks development is that all kinds of networks coexist. In addition, how to manage radio resource properly to meet the demands from users is a difficult issue. And an appropriate network selection method and resource allocation scheme is the effective approach for this problem.

In [1], the authors proposed a fitting factor based network selection algorithm. In that paper, the fitting factor combine several factors that relate to the network selection and user access to network with the biggest factor value. A location and mapping information based network selection algorithm is proposed in [2]. The authors in [2] take network load into consideration and update network condition information periodically. However the adoption of positioning system makes it more complex. In [3], the authors proposed an automatic vertical handoff algorithm that take into consideration of RSS, traffic contents, users' preferences and etc. [4] develops a network selection algorithm based on BP neutral algorithm and fuzzy logical. All the scheme mentioned above are single network selection. While the improvement of software and hardware technologies lead to the popularity of multi-mode terminals that equipped with multiple radio interfaces and can connect to several networks simultaneously. The authors in [5] proposed a utility based multi-service bandwidth allocation in HWNs where MMTs can simultaneously connect to several networks. A parallel transmission method adopting queueing theory is proposed in [6] for higher throughput. But both of the two papers don't take care of energy consumption on which individuals pay more and more attention.

In this paper, we put forward a TOPSIS based multi-radio access algorithm in heterogeneous wireless networks. MMTs determine candidate networks set according to RSS from available networks and active networks set based on TOPSIS method that considering energy consumption, throughput, cost and network load. Simulation results show that the proposed algorithm can enhance the throughput efficiently without consuming too much energy. 
The remainder of this paper is organized as follows. In Section 2, we introduce the heterogeneous wireless network model. The TOPSIS based multi-radio access networks selection algorithm in heterogeneous wireless networks is represent in Section 3. In Section 4, we evaluate the performance of the proposed algorithm and conclude the paper in Section 5.

\section{System model}

The heterogeneous wireless networks model is shown in Fig 1. It comprises Worldwide Interoperability for Microwave Access (WiMAX) networks, Universal Mobile Telecommunications System (UMTS) networks and Wireless Local Area Network (WLAN) access points. UMTS networks support wide coverage and user mobility but with low date rate. Compared with UMTS, WiMAX have higher date rate. WLAN provide high date rate and charge a low price, but are effectively only in hotspot areas. Users with traffic demand are in the whole area randomly.

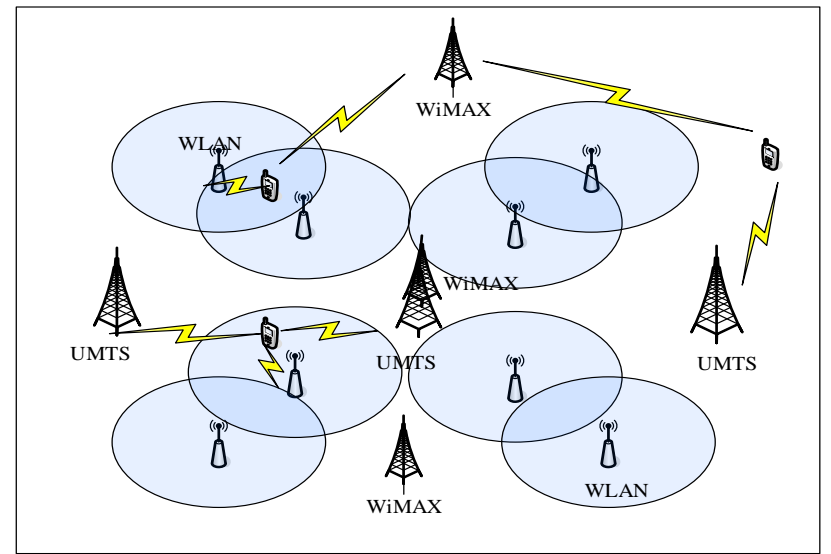

Fig.1. Heterogeneous wireless networks model

\section{TOPSIS based multi-radio access network selection}

The multi-mode terminals are equipped with various radio interfaces and an interface can only communicate to a network that match to this technology. In addition, all the interfaces can work simultaneously to enhance the data rate for users. In this paper, TOPSIS method is adopted to help each interface to select a proper network.

\section{Candidate networks determination.}

In order to assure normal communication with networks, the received signal strength from networks should no lower than RSS threshold. According to the channel propagation model [7], we take large scale fading and shadowing into consideration. The path loss of network $i$ at the distance $d$ is formulated by:

$$
P L_{i}(t)=P L_{i}^{0}+10 n_{i} \lg \left(d / d_{0}\right)+X(v, \delta)
$$

Where $d_{0}$ is the reference distance, $P L_{i}^{0}$ is the path loss of network $i$ at distance $d_{0}$. The path loss exponent $n_{i}$ of network $i$ is depend on wireless environment. Shadowing model $X(v, \delta)$ is a Gaussian random variable with mean $v$ and standard deviation $\delta$.

Assuming the transmission power of base station $i$ is $P_{i}^{\text {tbo }}$, the received signal strength of MT at the distance of $d$ can be obtained as follows:

$$
\operatorname{RSS}_{i}(t)=P_{i}^{t b 0}-P L_{i}(t)
$$

Canditate $=\left\{i \mid \operatorname{RSS}_{i}(t)>\operatorname{RSS}_{i}^{\text {th }}, i \in I\right\}$

Where Candidate represent the candidate network set and $I$ represents the total number in this network scenario.

\section{TOPSIS based network selection.}

After the candidate network selection mentioned above, it is supposed that there are $n 1$ UMTS networks, $n 2$ WIMAX networks and $n 3$ WLAN access points that comprise the UMTS 
candidate set $N_{1}$, WiMAX candidate set $N_{2}$ and WLAN candidate set $N_{3}$, respectively. Furthermore, we take $M$ attributes into consideration for each networks. Defining $A_{k}=\left[a_{i, j}\right]_{n_{k} * M}$ as the association matrix between user and networks, where $k=1,2,3$ represent the three kinds of networks, $a_{i, j}$ is the value of attribute $j$ in network $i$.

In general, the network attributes such as bandwidth, throughput and etc. are called beneficial attributes that the bigger value the better the attribute is and the cost attributes such as cost, delay and etc. are on the contrary. What's more, the normalization processing is necessary for the difference in terms of measurement unit and measuring method among various attributes. The normalized attribute metric is represented as $R_{k}=\left[r_{i, j}\right]_{n_{k} * M}$, where each element is defined as follows:

$$
r_{i, j}=\sqrt[a_{i, j}]{\sqrt{\sum_{i \in N_{k}} a_{i, j}^{2}}}
$$

Assuming that the attribute weight vector $W=\left[w_{1}, w_{2}, \ldots, w_{M}\right]$. The weighted normalized attribute metric combines the weight vector and normalized attribute metric formulated as $V_{k}=\left[v_{i, j}\right]_{n_{k} * M}$, where $v_{i, j}=r_{i, j} * w_{j}$ represents the weighted normalized attribute $j$ in network $i$.

In addition, the ideal scheme in TOPSIS method is the best attributes set and the negative ideal scheme is the worst attributes set as follows:

$$
\begin{aligned}
& S_{k}^{+}=\left[\left(\max _{i \in n_{k}} v_{i, j} \mid j \in J\right),\left(\min _{i \in n_{k}} v_{i, j} \mid j \in J^{\prime}\right)\right]=\left[v_{1}^{+}, v_{2}^{+}, \ldots, v_{M}^{+}\right] \\
& S_{k}^{-}=\left[\left(\min _{i \in n_{k}} v_{i, j} \mid j \in J\right),\left(\max _{i \in n_{k}} v_{i, j} \mid j \in J^{\prime}\right)\right]=\left[v_{1}^{-}, v_{2}^{-}, \ldots, v_{M}^{-}\right]
\end{aligned}
$$

Where $S_{k}^{+}$and $S_{k}^{-}$represent the ideal scheme and the negative ideal scheme, $J, J^{\prime}$ represent the beneficial attribute set and cost attributes set respectively.

The difference between evaluated solution and ideal scheme and negative ideal scheme are measured according to $M$-dimension Euclidean distance as follows:

$$
\begin{aligned}
& D_{i}^{+}=\sqrt{\sum_{j=1}^{M}\left(v_{i, j}-v_{j}^{+}\right)^{2}} \\
& D_{i}^{-}=\sqrt{\sum_{j=1}^{M}\left(v_{i, j}-v_{j}^{-}\right)^{2}}
\end{aligned}
$$

Based on the (7)-(8), the approaching degree of each network with ideal scheme can be expressed as follows and users access network with the biggest value:

$$
\begin{aligned}
& C_{i}=D_{i}^{-} /\left(D_{i}^{-}+D_{i}^{+}\right), i \in N_{k} \\
& O_{k}=\arg \max _{i \in N_{k}} C_{i}
\end{aligned}
$$

As mentioned above, MMTs access to the best performed network from each network subset and communicate with all the networks simultaneously which enhance the quality of service.

In this paper, we take of throughput, energy consumption, network load and cost into account.

Throughput: Based on Shannon expression and (2), the date rate can be obtained from network $i$ is given by:

$$
T h_{i}^{u}(t)=\rho_{i} b_{i}^{u} \log _{2}\left(1+\frac{R S S_{i}(t)}{N_{G}}\right)
$$

Where $\rho_{i}$ is throughput utilization ratio. The channel bandwidth acquired from network $i$ is expressed as $b_{i}^{u}$, and $N_{G}$ is the noise power in additive white Gaussian noise (AWGN) channel. 
Cost: The total expense depends on the bandwidth allocated from networks and network price. For the sake of simple, the cost model is given as:

$\cos t_{u}^{i}(t)=T h_{u}^{i}(t) * c_{i}$

Where $\cos t_{u}^{i}(t)$ is the total cost at time $t, c_{i}$ represent the price of network, $T h_{i}^{u}(t)$ represents the date rate obtained from network $i$ at time $t$.

Energy consumption: In spite of the benefit from multi-homing date transmission, using multiple radio interfaces lead to high energy consumption. How to make effective use of the limit batter energy of MMTs is studied in this work. In order to ensure a fixed received power $P_{i}^{r b 0}$ needed by network $i$, the transmission power $P_{u, i}^{t t}$ of MMT $u$ at the distance $d$ is derived as:

$$
P_{u, i}^{t t}(t)=P_{i}^{r b o}+P L_{i}(t)
$$

where the path loss $P L_{i}(t)$ is denoted in (1). Thus, given the fixed power consumption of transmitting and receiving $P C_{u, i}^{t t 0}$ and $P C_{u, i}^{r t o}$, the total power consumption is given by:

$$
P C_{u}(t)=\sum_{i} P C_{u}^{i}(t)=\sum_{i} P_{u, i}^{t t}(t)+P C_{u, i}^{t t 0}+P C_{u, i}^{r t 0}
$$

Load cost: The network load condition reflects the occupation situation of network resource and relate to the QoS directly. Networks can not support users well in heavy load condition. Thus in this paper, the load cost is defined.

$$
L_{i}=\left(\sum_{u} b_{i}^{u} / B_{i}\right)^{2}
$$

The throughput mentioned above belongs to beneficial attribute and the other three are cost attributes. The MMTs select a best performed network in each kind of radio access technology.

The algorithm consists of the following steps:

(1) MMTs determine the candidate networks set according to RSS.

(2) MMTs classify the candidate networks based on the radio access technology adopted and remove the networks that can't match with any interface of MMTs.

(3) MMTs select an optimal network from each kind of RAT according to TOPSIS method.

(4) MMTs connect to multiple networks simultaneously.

\section{Simulation results and discussion}

We evaluate the performance of the proposed algorithm in this paper by simulation with software MATLAB 2012a. There are three UMTS networks, three WiMAX networks and eight WLANs located in the area as shown in Fig.1. In this heterogeneous wireless networks scenario, users with multi-model terminal arrive in a Poisson process with parameter mean arrival rate $\xi$ user/s and each user arrival with traffic demand. The session holding time is exponentially distributed with mean $\psi$ s. The resource allocation algorithm will be initiated once user arrive, and doesn't change during the holding time. Assuming that all the RATs in the scenario work on different frequency band almost interference-free. The partial parameters of RATs are shown in Table 1[8].

Table 1 RAT parameters [8]

\begin{tabular}{|l|c|c|c|c|c|c|c|}
\hline Parameters & UMTS1 & UMTS2 & UMTS3 & WiMAX1 & WiMAX2 & WiMAX3 & WLAN1-8 \\
\hline BS location & $(0,0)$ & $(-800,0)$ & $(800,0)$ & $(0,0)$ & $(0,-800)$ & $(0,800)$ & random \\
\hline Cost(RMB/Mbps) & 1.00 & 1.03 & 1.05 & 0.82 & 0.84 & 0.85 & $0.41 \sim 0.43$ \\
\hline Coverage (m) & 3000 & 3000 & 3000 & 1500 & 1500 & 1500 & 100 \\
\hline Bandwidth (MHz) & 5 & 5 & 5 & 15 & 15 & 15 & 11 \\
\hline
\end{tabular}


The parameters of wireless channel propagation for simulation are shown in Table 2. And other parameters are set as follows, shadow fading with mean $v=0$ and standard deviation $\delta=3 d B$, noise power $N_{G}=4 * 10^{-11} \mathrm{~W}$, reference distance $d_{0}=1 \mathrm{~m}$. The fixed receiving power and transmission power of each RAT are $[22,24,27] \mathrm{mW}$ and $[35,37,45] \mathrm{mW}$.

Table 2 Propagation model parameters [9]

\begin{tabular}{|c|c|c|c|}
\hline parameters & UMTS & WiMAX & WLAN \\
\hline$P_{i}^{b_{0}}(d B m)$ & 30 & 27 & 20 \\
\hline$n_{i}$ & 3.5 & 3 & 4 \\
\hline$\rho_{i}$ & 0.85 & 0.9 & 0.9 \\
\hline$R S S^{t h}(d B m)$ & -110 & -108 & -87 \\
\hline$P L_{i}^{0}(d B)$ & 5 & 27 & 8 \\
\hline$P_{i}^{r b o}(d B m)$ & -62 & -61 & -57 \\
\hline
\end{tabular}

Comparison between proposed algorithm and single network access is made to illustrate the performance. In single network access scheme, mobile terminals select the network with the highest score from all candidate networks.

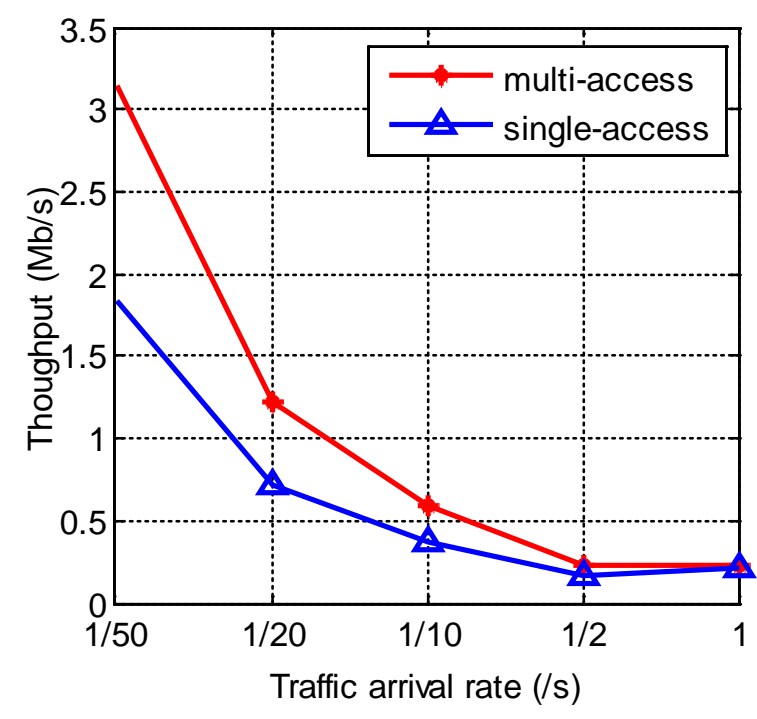

Fig.3. Throughput versus traffic arrival rate

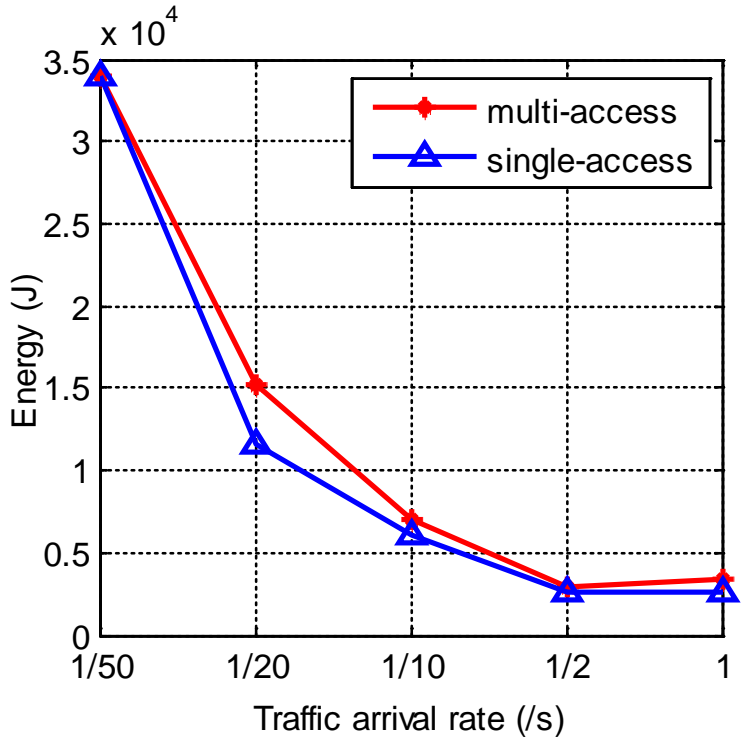

Fig.4. Energy versus traffic arrival rate

Fig 3 and Fig 4 shows the average throughput and average energy consumption for the two methods, respectively. In this paper, we assume the average holding time is $120 \mathrm{~s}$. It is observed that with the increase of the traffic arrive rate, the performance of throughput and energy are both decrease, especially at the beginning period. The reason is that the network resource reduce sharply with the increase of arrival of traffic and there is rarely resource for the latter traffic. However, it is observed that the proposed multi-radio access algorithm improves throughput at less 50 percent than single network access method in terms of throughput while it has little difference in terms of energy consumption.

\section{Conclusion}

In this paper, we put forward a TOPSIS based multi-radio access algorithm in heterogeneous wireless networks. MMTs determine candidate networks set according to RSS from available networks and active networks set based on TOPSIS method that considering networks load, cost, energy consumption and throughput. Simulation results show that the proposed algorithm can enhance the throughput efficiently without consuming too much energy. 


\section{Acknowledgements}

This work is supported by National Natural Science Foundation of China (61571234), National Basic Research Program of China (973 program: 2013CB329005), 863 Program (2014AA01A705), Graduate Student Innovation Plan of Jiangsu Province (SJLX15_0365)

\section{References}

[1] O. Sallent J. Perez-Romero, R. Ljung P. Karlsson, A. Barbaresi. Operator's RAT Selection Policies Based on the Fitting Factor Concept [C]. Mobile and Wireless Communications Summit, July, 2007 1-5.

[2] Junpei Kuboniwa, Yuji Miyake, Suguru Kameda, Akinori Tara, Hiroshi Oguma, Noriharu Suematsu, TadashiTakagi, Kazuo Tsubouchi. High Efficient Network Selection Scheme Using Location Information for Heterogeneous Wireless System [C]. Wireless Communications and Networking Conference Workshops, March 2015 9-12.

[3] Ajay Devendra Gurjar, Neetesh Purohit. An Optimized Network Selection Scheme for Heterogeneous Wireless Networks [C]. Sixth International Conference on Contemporary Computing, Aug, 2013 8-10.

[4] Jilei Yan, Linjing Zhao, Jiandong Li, Yingying Wang. A Network Selection Strategy in Resources Mobility environment[C]. Communications and Networking in China (CHINACOM), 2010 1-5.

[5] Changqing Luo, Hong Ji, Yi Li. Utility-based Multi-service Bandwidth Allocation in the 4G Heterogeneous Wireless Access Networks[C]. WCNC, 2009.

[6] Alessandro Bazzi, Gianni Pasolini, Oreste Andrisano. Multiradio Resource Management: Parallel Transmission for Higher Throughput?[J]. EURASIP Journal Advances in Signal Processing, 2008.

[7] LIU Chunyang, ZHU Qi. Bandwidth allocation scheme over wireless video communication [J]. Signal Processing, 2015, 31 (6) 737-743.

[8] Lina ZHANG, Qi ZHU, Shasha ZHAO. Multi-Access Selection Algorithm Based on Joint Utility Optimization for the Fusion of Heterogeneous Wireless Networks [J]. IEICE TRANSACTIONS on Communications, November, 2014, 97 (11) 2269-2277.

[9] Fan wenhao, Liu yuanan, Wufan. A terminal-controlled network access selection scheme for multi-radio access networks[J]. Journal of communications, 7, 2012, 33 (7) 183-190. 